\title{
The Lehigh Valley INTERACT: An operant research computer system for the noncomputer jockey*
}

\author{
ALAN C. KAMIL and ROBERT A. SACKS \\ University of Massachusetts, Amherst, Massachusetts 01002
}

The Lehigh Valley Electronics INTERACT computer system is a control and data-collection device which we have found well suited to a wide variety of animal operant experiments. The most impressive feature of this system is that it is very easy to use. Set-up is rapid, the ACT programming language can be learned quickly, and interfacing to operant chambers is straightforward. Initial set-up took only a few hours (mostly carrying the system up two flights of stairs). The inevitable software bugs (ours was the first NOVA-based system sold) took only 6-8 weeks to correct and are unlikely to plague future users. Within 1 week, both of us were fully able to program the system. Interfacing required only as much time as necessary to wire some cables, since the system is ready to be connected directly to operant chambers. In this paper, we will present a physical description of the INTERACT system we have been using (it is available in several different configurations), a description of the ACT language, and a summary of some of the ways in which we have used INTERACT. Finally, we will make some general comments on the relative strengths and weaknesses of this system and offer some general advice to the prospective purchaser.

\section{PHYSICAL DESCRIPTION}

The INTERACT system in use at the University of Massachusetts at Amherst is based upon a Data General NOVA computer (1200 series) with $8 \mathrm{~K}$ of core memory. The system includes two ASR-33 Teletypes, one for the input of systems commands, programs, and parameter changes and the other for data output. The system also includes three stations (up to eight can be used). Each station has interfacing consisting of $11-28-\mathrm{V} d c$ outputs and 11 response inputs suitable for direct connection to one or more operant chambers. A

* The research reported in this paper and preparation of the manuscript was supported in part by funds from the Graduate Research Council, University of Massachusetts, Amberst, by Training Grant MH-11823 from the National Institutes of Health, and by NSF Grant GB-30501 to the first author. high-speed optical paper-tape reader was added for the rapid loading of the ACT language and compiled programs. This reader is also useful for loading BASIC, which can be used for off-line data analysis.

The time-sharing capacity of the system allows the stations to function simultaneously and independently providing the single data-output device is not called by more than one station at a time. This output limitation has proved to be only a minor annoyance, since it can be easily overcome by making data output contingent upon an E-produced switch closure. Since any station can be used to control more than one chamber, the total number that can be controlled by an INTERACT system seems limited only by the available core memory and by the number of inputs and outputs on each station. However, relay or solid-state decoders can be used to increase the effective number of inputs and outputs of any station.

The system comes equipped with a 10-msec clock and a probability randomizer. Provision is made for disabling the peripherals in order to use the computer alone. No one in our laboratory has found it either necessary or desirable to learn the NOVA Assembly language, and the extensive Data General software included with the system is simply gathering dust.

\section{THE ACT PROGRAMMING LANGUAGE}

ACT (Automated Contingency Translator) is the programming language of the INTERACT system, explicitly written for operant research applications. The 11 outputs on each station are numbered in octal-1, 2, 4, $10,20,40,100,200,400,1000$, 2000. Each manipulandum is connected directly to 1 of the 11 station inputs which are numbered in the same way. Any output or combination of outputs can be specified by simply declaring a "state" while writing a program. States are declared by typing the letter $S$ followed by the number(s) of the desired state on the Teletype keyboard. Then, when that point in the program is reached during the experiment, $-28 \mathrm{~V} \mathrm{dc}$ will be provided at the declared outputs. For example, declaring "S4" will turn on Output 4; declaring "S46" will turn on Outputs 40, 4, and 2. A programmer can also specify "dummy states" such as "SO" which do not produce any output but are otherwise treated as valid states by the system. These dummy states are often useful for intervals in which all stimuli are off during an experiment or for states whose purpose is recording of data only. In addition, it is possible to declare states which are identical at the interface but are otherwise treated as distinct by INTERACT. "S4.10" and "S4.11" would both produce Output 4 at the station but would be treated as independent by the system. This feature is often useful for data collection as well as for situations in which different contingencies may be in effect at different times in the same stimulus complex. More than one state can be specified at a time, using a hierarchical or "nested" arrangement, indicated on the Teletype by indenting.

Transitions from one state to another can be made on three bases: (1) Elapsed time. The format for this type of transition is "After $10 \mathrm{sec}$ go to S2." The time interval can be expressed in hundredths of a second ("units"), seconds, minutes, or hours. (2) The occurrence of a specified number of responses. The format for this type of transition is "If $10 \mathrm{R} 1 \mathrm{go}$ to $54 . "(3)$ A change in the value of a variable relative to some predetermined value. The format here is "For $(\mathrm{HO})=31$ go to S100." There are 64 variables available per station. Variables may be initialized, incremented, or decremented any time a state is entered. This also may be accomplished at any time on the Teletype keyboard.

The example shown in Table 1 may help clarify this description of the ACT language. This program is a fixed-interval 20-sec fixed-ratio 15 multiple schedule in which the reinforcement contingency in effect is signaled by a cue. With this program, the $S$ would receive alternating single reinforcement periods of FI $20 \mathrm{sec}$ and FR 15. After 25 reinforcements, the program would stop and indicate that it had terminated by printing DONE on the output Teletype. After five R2000-five E-produced switch closures routed to Input 2000 on the interface-the data would be printed, and five more R2000 would restart the program. As the program is written, the data output would be the total number of nonreinforced responses during the FI segment and the total amount of time spent in the FR segment. By the use of different available programming techniques, additional data could be collected. For 
Program

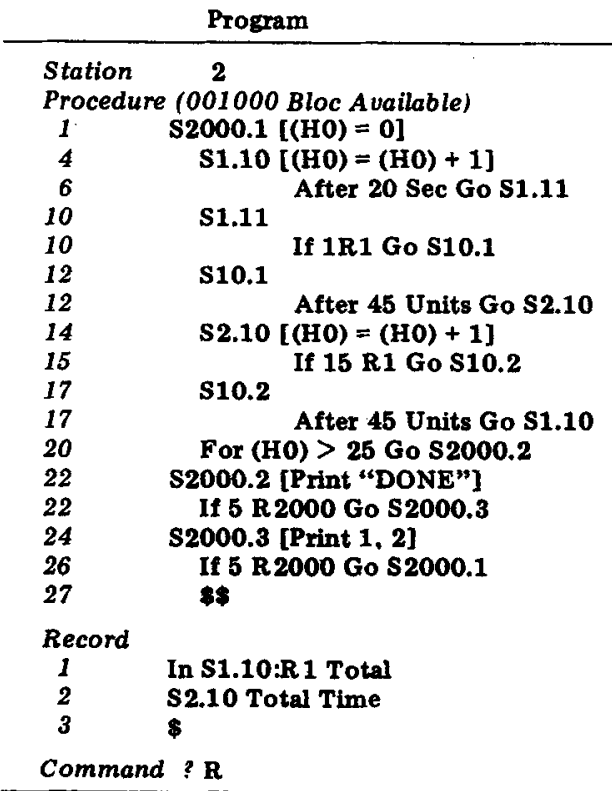

Descriptive Comments

Specifies station to which program applies

Specifies space available for program (octal)

Houselight on and initialize the variable (HO) at 0

Cue No. 1 for FI on and increment (HO)

Transition to next state, $\mathbf{S 1 . 1 1}$ based on elapsed time

Reinforcement set up, Cue 1 still on

Transition, based on response

Reinforcement

Transition, 45 units $=0.45 \mathrm{sec}$

Cue No. 2 for FR and increment (HO)

Transition based on number of responses

Reinforcement

Transition, loops back to start of FI component

Transition based on variable, exits S2000.1

Holding state at end of session, DONE printed on Teletype

Transition, R2000 an experimenter-produced switch closure

Holding state, data of Record Lines 1 and 2 outputted

Transition, restarts program

Indicates termination of program

Start of record section

Record number of responses during $\$ 1.10$

Record total duration of $\$ 2.10$ (in units)

Indicates termination of record section

Command starts program

*Italicized statements are printed by System, others by the Programmer

example, it would be quite simple to collect trial-by-trial printouts of latencies, interresponse times, etc. By using the probability randomizer, VI or VR schedules would be possible, as well as random orders of presentation of the schedules rather than simple alternation.

Only raw data can be retrieved from any experiment. There is no provision for any data reduction. Data are outputted as integers and can be formatted with the use of literals. A datum can either be printed on the Teletype (or punched on paper tape) when generated or stored for later output. Data available for output include the number of responses emitted in a state, the amount of time (in hundredths of a second) spent in any state, or the value of a variable.

Finally, there are several features of ACT which can be seen in Table 1 which relate to the writing of programs. Italicized statements in the table were written by the computer. When an $E$ begins to program, he is first queried as to which station will be used. Once he specifies a station, his program will operate only that station. INTERACT then informs the $E$ how many programming blocs are available (with our $8 \mathrm{~K}$ machine, with some space set aside for data storage and for the ACT language itself, we have $1000_{8}$ blocs available). The left-hand column of numbers printed out on each line is then indicative of how many programming blocs have been used to that point in the program. The available programming blocs can be apportioned among the stations in any desired way. The system compiles programs as they are written, line by line. Consequently, there is no editor. At the end of the program, signaled by a $\$ \$$ by the programmer, the computer then asks what is to be recorded. These data are called for printout by their line number in the record section. Literals are printed by the command PRINT followed by the literal in quotes.

\section{APPLICATIONS}

Hopefully, the above short course in ACT has given the reader some idea of the simplicity and flexibility of the INTERACT system in operant research. We have taken advantage of this flexibility by conducting a wide variety of experiments with the system over the past 11 months, often running as many as three simultaneously. The list below is a partial one but should help indicate the potential of INTERACT.

(1) Matching to sample (MTS): The MTS program in use in our laboratory controls two operant chambers (Lehigh Valley Electronics No. 132-02) simultaneously and independently on a single station with the external use of two relay decoders. Each chamber contains three pecking keys, each with a multiple stimulus projector mounted behind it, and a grain feeder. Our INTERACT MTS program is written such that the $E$ can, at any time, change the duration of reinforcement or time-out, change the number of responses required on any key, control the interval between the offset of the sample stimulus and the onset of the comparison stimuli, etc., by simply changing the value of variables on the Teletype keyboard. In addition, with some very simple external switching, the same program can be used with any of the 12 stimuli available on each stimulus projector and for nonmatching (oddity) as well as matching. This single program can thus be used to run a number of different experiments. One special feature of this program is the way in which it balances stimulus configurations. There are four stimulus configurations possible on any given trial. The system generates sets of 16 trials which consist of four of each possible configuration. At the outset of each block of 16 trials, each of the four possible configurations may occur with equal probability. Each time a given configuration is presented, its probability of reoccurrence is decremented. On the last trial of a 16-trial block, only one configuration can occur, the probability of all others having been reduced to zero. All probabilities are then reinitialized and a new 16-trial sequence is begun. Data recording includes the number correct on each configuration, which is printed out at the end of the session, along with a variety of other response measures, such as total number correct, number of responses on each of the three keys, etc.

(2) Learning set in bluejays: In this experiment, the INTERACT system controls a three-key Skinner box with a Davis Universal feeder. Choice stimuli are presented by a Carousel projector on the two side keys. A 
response to the center key starts each trial. The system is programmed to present 10 trials on sach of five two-choice discrimination problems in each session (these values are controlled by the values of variables and thus can be easily changed). Position of the correct stimulus is randomized, and new problems are presented by advancing the projector to a new pair of slides. At present, the number of responses required on the side keys is being varied. After each trial, the system prints the side correct, the side of the first response, whether or not the final response was correct or incorrect, the number of responses made on each key, and the latency of the choice response. This experiment has been programmed both with relay equipment, before we purchased the INTERACT system, and with INTERACT. It was much easier to program with the computer system, and it runs more reliably and with much greater flexibility now than it ever did, or could, with relay equipment.

(3) Multiple Sidman avoidance: This is an experiment programmed by John J. B. Ayres, who shares the INTERACT system with us. In this program, different responses are required to delay shocks in different components of the schedule. Response-shock and shock-shock intervals can be varied in each component. Two boxes are independently controlled by a single station. Data recording is extensive, including temporal distributions of responses in $2-\mathrm{sec}$ intervals, number of responses during various periods including during shock, number of shocks received, and so on.

In addition to the programs outlined above, we have also used INTERACT to control a variety of other experiments, including simple two-choice simultaneous discrimination and successive discrimination in rats, simple vs choice reaction time in humans, and simple schedules of reward. We have not yet even come close to an experimental situation we wished to program, but could not because of limitations in the flexibility of the system. It should not be implied from this that such limitations do not exist; they undoubtedly do. However, they seem unlikely to arise in most operant conditioning labs.

\section{SUMMARY}

The major advantages of INTERACT are that it provides a computer control system which is easy to learn, easy to set up and interface to typical experimental chambers, and essentially allows one to obtain many of the benefits of computer control without the necessity of spending considerable amounts of time becoming a computer jockey. Furthermore, we have always found the service offered by Lehigh Valley excellent, whether our need was for some help in figuring out how to program some experiment or the debugging of some problem which popped up.

The major disadvantages that we see are that the system is expensive, it undoubtedly is less flexible than a computer whose machine language the operator can speak (although INTERACT is surely easier to use), and it is a kind of "eggs in one basket" situation. If the system breaks down, everything stops. However, we have had minimal down-time problems. Finally, the ratio of memory needed per experiment is relatively high, primarily because the ACT language takes a lot of core (about 3.2K).

To the prospective purchaser, we would make the following general recommendations: (1) A minimum of $8 \mathrm{~K}$ core memory is necessary. If many stations, or highly complex experiments are planned, $12-16 \mathrm{~K}$ is advisable. (2) A high-speed reader is a necessity for the quick loading of ACT and already compiled programs. The low-speed readers on the ASR-33 Teletypes are slow and sometimes unreliable. (3) A high-speed punch may be advisable if data output will be considerable. (4) We have found the two-Teletype system advantageous, since it allows us to input new programs, etc., while data is being printed. Further, since it is very easy to switch the Teletypes, this set-up effectively gives us a spare Teletype in case of breakdown in either one. 